\title{
High order harmonic generation in noble gases using plasmonic field enhancement
}

\author{
M. F. Ciappina ${ }^{1 *}$, T. Shaaran ${ }^{1}$, and M. Lewenstein ${ }^{1,2}$ \\ ${ }^{1}$ ICFO-Institut de Ciènces Fotòniques, 08860 Castelldefels (Barcelona), Spain and \\ ${ }^{2}$ ICREA-Institució Catalana de Recerca i Estudis Avançats, Lluis Companys 23, 08010 Barcelona, Spain
}

\begin{abstract}
We present theoretical studies of high-order harmonic generation (HHG) in rare gases driven by plasmonic field enhancement. This kind of fields appears when plasmonic nanostructures are illuminated by an intense few-cycle laser and have a particular spatial dependency, depending on the geometrical shape of the nanostructure. We demonstrate that the strong nonhomogeneous character of the laser enhanced field plays an important role in the HHG process and significantly extends the harmonic cutoff. Our models are based on numerical solution of the time dependent Schrödinger equation (TDSE) and supported by classical and semiclassical calculations.
\end{abstract}

PACS numbers: 42.65.Ky,78.67.Bf, 32.80.Rm

\section{INTRODUCTION}

One of the most prominent demonstrations of the nonlinear character of the interaction of a strong laser with matter is high-order harmonic generation (HHG) 1, 2] . In the past two decades, this phenomenon has been an important subject in both theoretical and experimental physics, as a reliable tool for generating coherent light sources in the ultraviolet (UV) to extreme ultraviolet (XUV) spectral range. HHG based coherent light sources open the possibility for extracting temporal and spatial information with attosecond and sub-Angstrom resolution [3]. As a results, HHG has found numerous applications in basic research, biology, material science, and even lithography [4].

The physical mechanism behind HHG can be understood by a semi-classical three-step model [5, 6]. According to this model, in the first step, an electron reaches the continuum with no kinetic energy through tunneling ionization. In the second step, the released electron subsequently accelerated in an oscillating electric field until it is driven back by the laser field toward its parent ion or molecule. In the third step, upon its return, it recombines with the core and emits a high-energy photon, with energy equal to the sum of the electron kinetic energy and the ionization potential. In this model, the propagation is treated classically while the ionization and recombination processes are described quantum mechanically. The maximum photon energy (cutoff) producible with high harmonic generation is given by $I_{p}+3.17 U_{p}$, where $I_{p}$ is the atomic ionization potential, while $U_{p}=I / 4 \omega^{2}$ is the ponderomotive energy in the laser field of intensity $I$ and frequency $\omega$.

For high-order harmonic generation to occur, one needs a laser field with intensity greater than $10^{13} \mathrm{~W} / \mathrm{cm}^{2}$ ), two orders of magnitude larger than the output of the current modest femtosecond oscillator. It means an additional process like chirped-pulse amplification is needed to reach

\footnotetext{
*Present address: Department of Physics, Auburn University,
} Auburn, AL 36849, USA the required intensity for generation of high harmonics using noble gases. Even so, the XUV based on HHG has low duty cycle and efficiency [7].

The recent demonstration based on surface plasmon resonances as light amplifiers could overcome these difficulties [7, 8]. By maneuvering surface plasmon resonances, the laser electric fields can locally be enhanced by more than $20 \mathrm{~dB}$ [9, 10] without the need of extra cavities or laser pumping process. Indeed, in such a system the intensity of the locally enhanced field become large enough to exceed the threshold intensity for harmonic generation in noble gases. In particular, in the case of gold bow-tie-shaped nanostructures, the initial laser field (800 nm laser with intensity $10^{11} \mathrm{~W} / \mathrm{cm}^{2}$ ) enhanced sufficiently to generate the 7 th $(114 \mathrm{~nm})$ to the 17st $(47 \mathrm{~nm})$ harmonics. Moreover, each nanostructure could potentially act as a point-like source to generate high harmonic radiation. This would allow collimation of these coherent radiations by means of constructive interference. This would open a wide range of possibilities to spatially rearrange nanostructures to enhance and even shape the HHG spectra [7]. Additionally, one of the most unwanted effects of the harmonic propagation, namely the phase matching, appears to be not important at all in the HHG using plasmonic fields [8].

The physical mechanism of HHG based on plasmonics can be explained as follows (for full explanation see [7]). A femtosecond low intensity laser pulse couples to the plasmon mode and initiates a collective oscillation among the free charges within the metal. This causes a large resonant enhancement of the local field inside and at the nanostructure vicinity. This enhancement is well above the threshold intensity for generating high harmonics. Consequently, by injecting rare gases into the site of the enhanced field HHG can be produced. In here, the enhanced field is spatially inhomogeneous in the region where electron dynamics take place. In addition, the movement of the electron in the enhanced field is restricted in space. These two features imply strong modifications in the harmonic spectra, as was shown by several authors [11 14]. Furthermore, the influence of the nonhomogeneous character of the laser electric field in the photoelectron spectra was recently assessed [15]. 
The outcome of Kim et al. 7] experiment, however, has been recently under intense scrutiny [16 18]. Between the points to elucidate are the real intensity enhancement of the input laser field and the damage threshold of the gold bow-tie nanostructures. According to the finiteelement simulations presented in Ref. [7], the local field intensity could be enhanced by 4 orders of magnitude ( 40 $\mathrm{dB}$ ). It means for input laser intensities of the order of $10^{11} \mathrm{~W} \mathrm{~cm}^{-2}$ the enhanced field intensity could reach to the order of $10^{15} \mathrm{~W} \mathrm{~cm}^{-2}$ in the vicinity of the bow-tie nanostructure. On the other hand, from the measured high-order harmonic spectra it appears that these numbers are unrealistic. The highest measured harmonic was $n \approx 17$, a limit corresponds to intensities of the order of $5 \times 10^{13} \mathrm{~W} \mathrm{~cm}^{-2}$ (we have estimated this value using the well established three step or simple man's model [6]). It is clear that the measured value for enhanced field intensity is different from the ones obtained in the finiteelement simulations of Ref. 7]. In our theoretical models we have considered this point using a reduction factor in the field enhancement obtained from the finite element simulations. Moreover, for this work we use a laser pulse with a longer wavelength, in order to have larger electron excursion, to explore the effect of the field nonhomogeneity more clearly.

To our best knowledge, apart from Ref. [7] there is only one more experiment in which HHG was produced from noble gas using metallic nanostructures [8]. Recently, however, different kinds of nanostructures, e.g. nanoparticles or nanotips, have been used to generate high energy electrons directly from the nanostructure in the absence of the gas and to study the distinct and new characteristics of these nanosytems (see e.g. [19 23] )

The paper is organized as follows. In the next section we present a numerical calculation of HHG by incorporating the actual functional form of the electric field in the vicinity of a metal bow-tie shaped nanostructure in the time dependent Schrödinger Equation. A classical and semiclassical analysis of an approximated model for nonhomogeneous fields is presented in Section 3. We finish with our conclusions and a brief outlook.

\section{SINGLE ATOM RESPONSE OF PLASMONIC ENHANCED FIELDS}

Most of the numerical and semiclassical approaches to study laser-matter processes in atoms and molecules, in particular high-order harmonic generation (HHG), are mainly based on the dipole approximation in which the laser electric field $(\mathbf{E}(\mathbf{r}, t))$ and its vector potential associated $(\mathbf{A}(\mathbf{r}, t))$ are spatially homogeneous in the region where the electron dynamics takes place, i.e. $\mathbf{E}(\mathbf{r}, t)=\mathbf{E}(t)$ and $\mathbf{A}(\mathbf{r}, t)=\mathbf{A}(t)[1,2]$. On the other hand, the fields generated using plasmonics nanosystems, such as metallic nanoparticles, nanotips and gold bowtie shape nanostructures, present a strong spatial dependence, which cannot be ignored. From a theoretical viewpoint, the HHG process using homogeneous fields can be tackled using different approaches and approximations (for a summary see e.g. 24, 25] and references therein). In this section we employ the Time Dependent Schrödinger Equation (TDSE) in order to study the harmonic radiation generated by a model atom when it is illuminated by a spatially inhomogeneous electric field. This in an actual field, which is obtained directly from 3D finite-element (FE) simulations where the real parameters of the bow-tie shaped nanostructures, as those used in [7], are considered. For linearly polarized fields, which is the case of our study, the dynamics of an atomic electron is mainly along the direction of the laser electric field and as a result it is a good approximation to employ the Schrödinger equation in one spatial dimension (1D-TDSE) [1] which can be written as follows:

$$
\begin{aligned}
\mathrm{i} \frac{\partial \Psi(x, t)}{\partial t} & =\mathcal{H}(t) \Psi(x, t) \\
& =\left[-\frac{1}{2} \frac{\partial^{2}}{\partial x^{2}}+V_{A}(x)+V_{L}(x, t)\right] \Psi(x, t)
\end{aligned}
$$

where $V_{A}(x)$ is the atomic potential and $V_{L}(x, t)$ represents the potential due to the laser electric field. In here, we use for $V_{\text {atom }}$ the "quasi-Coulomb" or "soft-core" potential

$$
V_{A}(x)=-\frac{1}{\sqrt{x^{2}+\xi^{2}}}
$$

which first was introduced in [26] and since then has been widely used in the reduced dimensions studies of lasermatter processes in atoms. The required ionization potential can be defined by varying the parameter $\xi$ in Eq. (2). The laser potential $V_{L}(x, t)$ of the laser electric field $E(x, t)$ is given by

$$
V_{L}(x, t)=E(x, t) x .
$$

In Eq. (3), the spatial dependency of $E(x, t)$ can be defined in terms of a perturbation to the dipole approximation and it reads

$$
E(x, t)=E_{0} f(t)(1+h(x)) \sin \omega t,
$$

which is linearly polarized along the $x$-axis. In Eq. (4), $E_{0}, \omega$ and $f(t)$ are the peak amplitude, the frequency of the coherent electromagnetic radiation and the pulse envelope, respectively. In addition, $h(x)$ represents the functional form of the nonhomogeneous electric field and it can be written as a power series of the form $h(x)=$ $\sum_{i=1}^{N} b_{i} x^{i}$. The coefficients $b_{i}$ are obtained by fitting the actual electric field that results from a $\mathrm{FE}$ simulation considering the real geometry of different nanostructures (See panel (b) of Fig. 1). In this work we use for the short laser pulse a trapezoidal envelope given by

$$
f(t)= \begin{cases}\frac{t}{t_{1}} & \text { for } 0 \leq t<t_{1} \\ 1 & \text { for } t_{1} \leq t \leq t_{2} \\ -\frac{\left(t-t_{3}\right)}{\left(t_{3}-t_{2}\right)} & \text { for } t_{2}<t \leq t_{3} \\ 0 & \text { elsewhere }\end{cases}
$$


where $t_{1}=2 \pi n_{\text {on }} / \omega, t_{2}=t_{1}+2 \pi n_{p} / \omega$, and $t_{3}=t_{2}+$ $2 \pi n_{o f f} / \omega \cdot n_{o n}, n_{p}$ and $n_{o f f}$ are the number of cycles of turn on, plateau and turn off, respectively.

We employ $\xi=1.18$ in Eq. (2) such that the binding energy of the ground state of the 1D Hamiltonian coincides with the (negative) ionization potential of Ar, i.e. $\mathcal{E}_{G S}=-15.7596 \mathrm{eV}$ (-0.58 a.u.). Furthermore we assume that the noble gas atom is in its initial state (ground state (GS)) before we turning the laser $(t=-\infty)$ on. Equation (10) is solved numerically by using the CrankNicolson scheme [1]. In addition, to avoid spurious reflections from the spatial boundaries, at each time step, the electron wave function is multiplied by a mask function [27].

The harmonic yield of an atom or molecule is proportional, in the single active electron approximation, to the Fourier transform of the acceleration $a(t)$ of the active electron [28]. That is,

$$
D(\omega)=\left|\frac{1}{\tau} \frac{1}{\omega^{2}} \int_{-\infty}^{\infty} \mathrm{d} t \mathrm{e}^{-\mathrm{i} \omega t} a(t)\right|^{2}
$$

with $a(t)$ is obtained by using the following commutator relation

$$
a(t)=\frac{\mathrm{d}^{2}\langle x\rangle}{\mathrm{d} t^{2}}=-\langle\Psi(t)|[\mathcal{H}(t),[\mathcal{H}(t), x]]| \Psi(t)\rangle .
$$

In here, $\mathcal{H}(t)$ and $\Psi(x, t)$ are the Hamiltonian and the electron wave function defined in Eq. (11), respectively. The function $D(\omega)$ is called the dipole spectrum, which gives the spectral profile measured in HHG experiments. For solving Eq. (11), the gap size $g$ of the gold bow-tie nanostructure is taken into account restricting the spatial grid size (see Figure 1).

The electric field intensity distribution inside the gap of gold bow-tie shaped nanoantennas was computed numerically by 3D Finite Element Method (COMSOL Multiphysics) 29], using the gold optical properties taken from Ref. [30]. The nanoantenna is formed by two identical (isosceles) triangular gold pads (longest altitude of $600 \mathrm{~nm}$ and the smallest acute angle of $30^{\circ}$ ) separated by an air gap $g$. The apices at corners were rounded (10 $\mathrm{nm}$ radius of curvature) to account for limitation of current fabrication techniques and avoid nonphysical fields enhancement due to tip-effect. The out of plane thickness is set to $25 \mathrm{~nm}$. These parameters yield to a dipolar bonding resonance centered at around $\lambda=1800 \mathrm{~nm}$ when considering gaps ranging between $12 \mathrm{~nm}$ and 15 $\mathrm{nm}$. This particular value of $\lambda$ was chosen according to the availability of laser sources [31]. On the other hand, the selected laser wavelength allows the electron to have excursions of the order of the gap $g$ and consequently to confine its movement. Classically the electron excursion in an oscillating electric field is given by the so-called quiver radius $\alpha_{0}$, which is $\propto \sqrt{I} \lambda^{2}$ where $I$ is the laser intensity. For instance, in here, for intensities $I$ of $\sim 10^{14}$ $\mathrm{W} \mathrm{cm}{ }^{-2}, \alpha_{0}$ can have a value about \pm 80 a.u. $( \pm 4.5 \mathrm{~nm})$.

The panel (a) of Fig. 1 displays the calculated electric field enhancement in the gap of the bow-tie structures
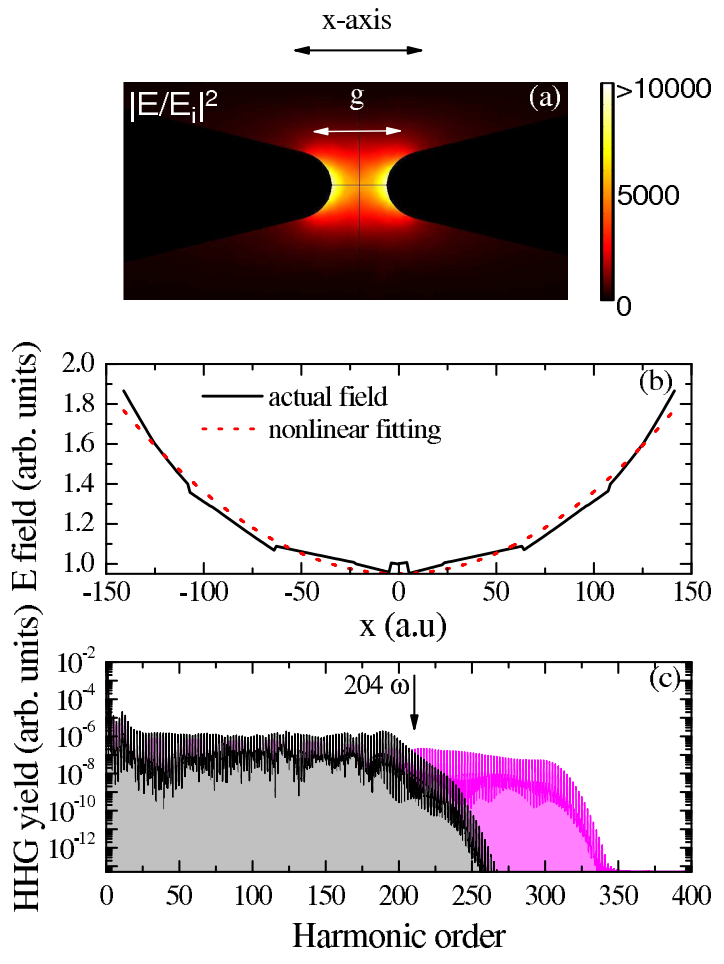

FIG. 1: (Color online) Panel (a) electric field enhancement in the gap region of the gold bow-tie nanostructure extracted from the FE simulations; panel (b) functional form of the electric field $E(x, t)$ where the solid lines is the raw data obtained from the FE simulations and the red dotted line is a nonlinear fitting; panel (c) high-order harmonic generation (HHG) spectra for Ar with ionization potential $\mathcal{E}_{G S}=-0.58$ a.u., laser wavelength $\lambda=1800 \mathrm{~nm}$ and intensity $I=1.25 \times 10^{14}$ $\mathrm{W} \cdot \mathrm{cm}^{-2}$ at the center of the gap $x=0$. We use a trapezoidal shaped pulse, Eq. (5), with $n_{o n}=3, n_{\text {off }}=3$ and $n_{p}=4$ (about $60 \mathrm{fs}$ ). The gold bow-tie nanostructure has a gap $g=15 \mathrm{~nm}$ (283 a.u.). Black line indicates the homogeneous case while magenta line indicates the nonhomogeneous case. The arrow indicates the cutoff predicted by the semiclassical model for the homogeneous case [6].

when illuminated by a linearly polarized ( $x$-axis) plane wave at $1800 \mathrm{~nm}$. The field-enhancement profile is extracted for the bow-tie long axis through the middle of the gap, so the successive problem is reduced to $1 \mathrm{D}$ as is shown in panel (b) of Fig. 1. Additionally we normalize the electric field by setting $E(0, t)=1$. We observe a typical amplification between $30-40 \mathrm{~dB}$, i. e. $3-4$ orders of magnitude between the input intensity and the intensity at center of the gap. In a real experiment, however, the enhancement can be smaller than our calculations; nevertheless, for a similar system, it was shown that one can obtain values of more than $20 \mathrm{~dB}$ [7, 8].

Panel (c) of Figure 1 depicts the harmonic spectra for a bow-tie shaped nanostructure with gap $g=15 \mathrm{~nm}$ and for a laser wavelength of $\lambda=1800 \mathrm{~nm}$ considering an homogeneous electric field, i.e $E(x, t)=E(t)$ and a nonhomogeneous electric field using Eq. (41). The enhanced laser intensity is $I=1.25 \times 10^{14} \mathrm{~W} \mathrm{~cm}^{-2}$ at the center of 
the spot $(x=0)$. In order to reach this intensity value, we consider plasmonic field enhancements between 25 and $35 \mathrm{~dB}$ with input intensities in the range of $4 \times 10^{11}$. $4 \times 10^{10} \mathrm{~W} \mathrm{~cm}^{-2}$. These intensities would be well below the damage threshold of the nanostructure employed (see e.g. 7]). In both cases, we use a trapezoidal shaped pulse with three optical cycles turn on $\left(n_{o n}=3\right)$ and turn off $\left(n_{o f f}=3\right)$ and a plateau with 4 optical cycles $\left(n_{p}=4\right)$, i.e. 10 optical cycles in total which is about $60 \mathrm{fs}$.

For the homogeneous case we have an harmonic cutoff at around $204 \omega$ as shown by the arrow in panel (c) of Fig. 1. In fact, our calculation are in excellent agreement with the semiclassical model [6]. For the nonhomogeneous case, however, we observe a substantial increase in the harmonic cutoff, which is about $50 \%$ higher than the cutoff generated by a homogeneous electric field. This new feature emerges due to the combination of the nonhomogeneous character of the electric field and the confinement of the electron motion [13]. In fact, the laser ionized electron could be absorbed by the metallic surface (for details see [11]) and in this way only the short electron trajectories would be allowed. This circumstance is comparable to the restriction in the electron motion incorporated in our theoretical model [32].

\section{CLASSICAL AND SEMICLASSICAL APPROACHES}

In this section we present classical and semiclassical studies of a particular case of nonhomogeneous field. In particular, we only consider the linear term of the series for $h(x)$ (see previous Section). This field represents, for instance, a first order approximation of the near-field in the surrounding of a metal nanoparticle [33, 34]. The main advantages of this approximation is the possibility to perform a quasi analytical approach using the Strong Field Approximation (SFA) [6]. In this way, the main features behind the quantum trajectories are elucidated and characterized.

\section{A. Strong Field and the Newton equation}

We use Eq (3) in order to calculate the Newton equation of motion of an electron in a nonhomogeneous electric field. In particular, the Newton equation of motion for an electron in this field is given by

$$
\ddot{x}(t)=-\nabla_{x} V_{L}=-x \nabla_{x} E_{e f f}(t, x)-E_{e f f}(t, x),
$$

where $E_{\text {eff }}(t, x)$ gives the effective electric field along the electron trajectory. For the homogeneous case, in which the laser field does not have spatially dependency and it is just $E(t)$, the Newton equation reads $\ddot{x}(t)=-E(t)$. On the other hand, if the spatial dependence of the enhanced laser electric field is perturbative and linear with respect to position, then the effective field can be approximated as

$$
E_{e f f}(t, x) \simeq E(t)(1+\alpha x)
$$

where $\alpha \ll 1$ is a parameter that characterize the strength of the inhomogeneity.

By substituting (9) into (8), we have

$$
\ddot{x}(t)=-E(t)(1+2 \alpha x(t))=-E(t, x) .
$$

Classically, the electron trajectory can be found by solving $\mathrm{Eq}$ (10). In here, we solve this equation by applying the Picard iteration 35] method and restrict ourselves to the first order (for more details see [13]). In the SFA, the action is defined in terms of the vector potential field $A(t)$, the counterpart of the velocity $\dot{x}(t)$ in the classical equation, which corresponds to the integration of $\ddot{x}(t)$ with respect to $t$. By assuming that the electron starts its movement from the origin with zero velocity, i.e. $x(0)=0$ and $\dot{x}(0)=0$, we will have

$$
x(t)=\lambda(t)-\lambda\left(t_{0}\right)-A\left(t_{0}\right)\left(t-t_{0}\right) .
$$

with $\lambda(t)=\int_{0}^{t} d t^{\prime} A\left(t^{\prime}\right)$. Furthermore, if we assume the potential field is zero at time $t_{0}$, then

$$
x(t)=\int^{t} d t^{\prime} A\left(t^{\prime}\right) .
$$

By using Eqs. (12) and (10), the effective vector potential along the electron trajectory $A_{e f f}(t, x)$ reads

$$
A_{e f f}(t)=A(t)+2 \alpha A_{a}(t)
$$

where

$$
A_{a}(t)=\int^{t} d t^{\prime \prime} A\left(t^{\prime \prime}\right)-\int^{t} d t^{\prime \prime} A^{2}\left(t^{\prime \prime}\right)
$$

\section{B. Transition Amplitude}

The strong field approximation (SFA) is based on the assumptions that the influence of the laser field is neglected when the electrons are bound to their target atoms and the binding ionic potential is neglected when the electrons are in the continuum. As a result, the free electrons in the continuum are described by field-dressed plane waves, which are known as Volkov states [36, 37.

Based on the well established model of Lewenstein [6], the SFA transition amplitude for HHG reads (in atomic units)

$$
\begin{aligned}
b_{\Omega}= & i \int_{-\infty}^{\infty} d t \int_{-\infty}^{t} d t^{\prime} \int d^{3} k d_{r e c}^{*}(\widetilde{\mathbf{k}}(t)) d_{i o n}\left(\widetilde{\mathbf{k}}\left(t^{\prime}\right)\right) \\
& e^{-i S\left(\Omega, \mathbf{k}, t, t^{\prime}\right)}+\text { c.c. }
\end{aligned}
$$


with the action

$$
\begin{aligned}
S\left(\Omega, \mathbf{k}, t, t^{\prime}\right)= & \int_{t^{\prime}}^{t} \frac{[\mathbf{k}+\mathbf{A}(\tau)]^{2}}{2} d \tau+I_{p}\left(t-t^{\prime}\right)-\Omega t+ \\
& 2 \alpha \int_{t^{\prime}}^{t} A_{a}(\tau)[\mathbf{k}+\mathbf{A}(\tau)] d \tau+ \\
& 2 \alpha^{2} \int_{t^{\prime}}^{t} A_{a}^{2}(\tau) d \tau
\end{aligned}
$$

and the prefactors

$$
\begin{gathered}
d_{\text {ion }}\left(\widetilde{\mathbf{k}}\left(t^{\prime}\right)\right)=\left\langle\tilde{\mathbf{k}}\left(t^{\prime}\right)\left|H_{\text {int }}\left(t^{\prime}\right)\right| \phi_{0}\right\rangle \\
d_{r e c}(\widetilde{\mathbf{k}}(t))=\left\langle\tilde{\mathbf{k}}(t)\left|O_{\text {dip }} \cdot e_{x}\right| \phi_{0}\right\rangle .
\end{gathered}
$$

In here, $I_{p}, k, \Omega, H_{\text {int }}\left(t^{\prime}\right), O_{\text {dip }}$ and $e_{x}$ denote the ionization potential of the field-free bound state $\left|\phi_{0}\right\rangle$, the drift momentum of the electron in the continuum, the harmonic frequency, the interaction of the system with the laser field, the dipole operator and the laser polarization vector, respectively.

Eq. (15) describes a physical process in which an electron, initially in a bound state $\left|\phi_{0}\right\rangle$ with energy $I_{p}$, interacts with the laser field by $H_{\text {int }}\left(t^{\prime}\right)$ at the time $t^{\prime}$ and ionized into a Volkov state $|\tilde{\mathbf{k}}(t)\rangle$. Subsequently, it propagates in the continuum from time $t^{\prime}$ to $t$. At the time $t$ it is driven back to the parents ion and recombines with the core emitting high-harmonic radiation of frequency $\Omega$. The SFA is not gauge invariant, thus, Eqs (17) and (18) will have different form in the length and velocity gauges. For the length gauge, the interaction Hamiltonian and the Volkov wave function are given by $H_{\text {int }}^{l}(t)=$ $r . E\left(t^{\prime}, x\right)$ and $\widetilde{\mathbf{k}}(t)=\mathbf{k}+A_{e f f}(t)$, respectively, while for the velocity gauge they are $H_{\text {int }}^{l}(t)=\left[k+A_{\text {eff }}\left(t^{\prime}\right)\right] / 2$ and $\widetilde{\mathbf{k}}(t)=\mathbf{k}$, respectively. In this work, we consider the length gauge and assume that the electric field is linearly polarized along $x$-axis. Furthermore, we consider an hydrogenic $1 s$ state for the field-free bound state $\left|\phi_{0}\right\rangle$.

As a result, Eqs. (17) and (18) yield

$$
\begin{gathered}
d_{i o n}\left(\widetilde{\mathbf{k}}\left(t^{\prime}\right)\right) \propto \frac{\widetilde{\mathbf{k}}\left(t^{\prime}\right)_{x}}{\left(\widetilde{\mathbf{k}}\left(t^{\prime}\right)^{2}+2 I_{p}\right)^{3}} E\left(t^{\prime}\right), \\
d_{r e c}(\widetilde{\mathbf{k}}(t)) \propto \frac{\widetilde{\mathbf{k}}(t)_{x}}{\left(\widetilde{\mathbf{k}}(t)^{2}+I_{p}\right)^{3}}
\end{gathered}
$$

respectively.

\section{Saddle-point equations}

The transition amplitude (15) was computed employing the saddle-point method [38]. For this approach one needs to obtain the saddle points for which the action (16) is stationary, i.e. for which $\partial_{t} S\left(\Omega, \mathbf{k}, t, t^{\prime}\right)=$ $\partial_{t^{\prime}} S\left(\Omega, \mathbf{k}, t, t^{\prime}\right)=\partial_{k} S\left(\Omega, \mathbf{k}, t, t^{\prime}\right)=0$. The solutions of the saddle point equations are directly related to the classical trajectories, which allows to demonstrate the cutoff of the HHG. The stationary conditions upon $t, t^{\prime}$ and $k$ lead to the saddle-point equations

$$
\begin{gathered}
{\left[\mathbf{k}+\mathbf{A}\left(t^{\prime}\right)\right]^{2}-2 \epsilon \beta\left(t^{\prime}\right)=-2 I_{p}} \\
\int_{t^{\prime}}^{t} d \tau[\mathbf{k}+\mathbf{A}(\tau)]+2 \alpha \eta(\tau)=0
\end{gathered}
$$

and

$$
\Omega=\frac{[\mathbf{k}+\mathbf{A}(t)]^{2}}{2}+I_{p}+2 \alpha \beta(t)
$$

with

$$
\beta(t)=A_{a}(t)[\mathbf{k}+\mathbf{A}(t)]+\alpha A_{a}^{2}(t),
$$

and

$$
\eta(\tau)=\int_{t^{\prime}}^{t} A_{a}(\tau) d \tau
$$

Eq. (21) gives the conservation law of energy for the electron tunnel ionized at the time $t^{\prime}$. Eq. (22) constrains the intermediate momentum of the electron as well as guarantees that the electron returns to its parent ion. Finally, (Eq. 23) expresses the energy conservation of the electron at the recombination time $t$ and generation of a high frequency photon $\Omega$.

The terms $\beta\left(t^{\prime}\right), \eta(t)$ and $\beta(t)$ in the Eqs. (21), (22), and (23), respectively, give the nonhomogeneous character of the laser field and they are zero for the homogeneous case $(\epsilon=0)$. For small $\epsilon$, the solutions of the saddle equations are generally complex since Eq. (21) has no real solutions, unless $I_{p} \rightarrow 0$. For nonhomogeneous field, $3.17 U_{p}$, where $U_{p}=E_{0}^{2} /\left(4 \omega^{2}\right)$ is the ponderomotive energy, does not directly corresponds to the maximum kinetic energy that the electron gains in the continuum . Depends on the nonhomogeneous character of the field, for the positive and negative $A_{a}$ this value is larger and smaller than $3.17 U_{p}$, respectively.

We now examine the drift momentum $\mathbf{k}$ of the electron at the time of the tunneling. For that, we take the limit in which the electron reaches the continuum with zero kinematical momentum, $I_{p} \rightarrow 0$. As a result, Eq. (21) reads

$$
\mathbf{k}=-A\left(t^{\prime}\right)+\epsilon A_{a}\left(t^{\prime}\right)(1 \mp \sqrt{3})
$$

In here, $\mathbf{k}$ has two different solutions with one lowering and the other exceeding and the homogeneous drift momenta. This result is different from the homogeneous case, in which $\mathbf{k}=-A\left(t^{\prime}\right)$. This deviation emerges from the strength of the inhomogeneity $\varepsilon$ and the shape of the $A_{a}\left(t^{\prime}\right)$. 


\section{Orbits}

In this section, we investigate the contribution of individual trajectories to the HHG cutoff of the homogeneous and nonhomogeneous cases by examining the solutions of the saddle point equations. To get a better insight into the problem, we employ a monochromatic field with $E(t)=E_{0} \sin (\omega t) e_{x}$, where $e_{x}$ is the polarization vector along the $x$ axis. By using the conventional relationship

$$
\mathbf{A}(t)=-\int_{-\infty}^{t} E\left(t^{\prime}\right) d t^{\prime}
$$

and applying some trigonometric identities, the electric field (10) and potential field (13) read

$$
\begin{aligned}
E(t) & =E_{0} \sin (\omega t)\left(1+2 \alpha \sin (\omega t) / \omega^{2}\right), \\
A_{e f f}(t) & =A_{0} \cos (\omega t)+2 \alpha A_{a}(t),
\end{aligned}
$$

respectively, where $A_{0}=E_{0} / \omega$ and

$$
A_{a}(t)=A_{0}^{2} \sin (\omega t) / 4 \omega-A_{0}^{2} t / 2
$$

In terms of the pondermotive energy of the homogeneous field, the drift momentum of Eq. (26) yields

$$
\mathbf{k}=-2 \sqrt{U_{p}} \cos \left(\omega t^{\prime}\right)+\epsilon\left[\frac{U_{p}}{\omega} \sin \left(\omega t^{\prime}\right)-2 U_{p} t^{\prime}\right](1 \mp \sqrt{3})
$$

Based on the above equations, we solve the saddle point equations defined in Eqs. (21) - (23) in terms of the ionization $\left(t^{\prime}\right)$ and recombination $(t)$ times.

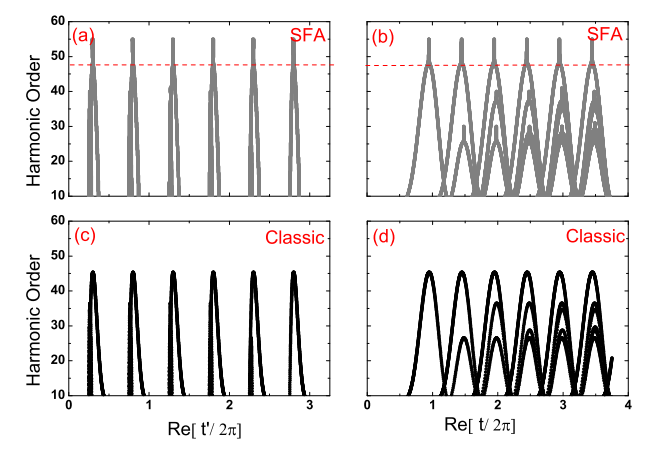

FIG. 2: (Color online) Dependence of harmonic order on the release time $t^{\prime}$ and the recombination time $t$ of the electron for the homogeneous field $(\varepsilon=0)$. We consider an hydrogen atom, for which the ground-state energy is $I_{p}=0.5$ a.u., in a linearly polarized, monochromatic field of frequency $\omega=0.057$ a.u. and intensity $I=3 \times 10^{14} \mathrm{~W} / \mathrm{cm}^{2}$. Panels (a) and (b) give the harmonic order as a function of the ionization and recombination times of the SFA model, respectively, while panels (c) and (d) depict the the harmonic order in terms of ionization and recombination times of the classical calculations, respectively. The red dashed lines correspond to the harmonic cutoff.
In this paper, we present these solutions briefly, for more detailed analysis we refer to our previous paper [39]. In Figs. 2] and 3, we plot the harmonic order as function of the real parts of the ionization $\left(t^{\prime}\right)$ and recombination $(t)$ times for the case with $\varepsilon=0$ and $\varepsilon=0.003$ (panels $\mathrm{a}$ and $\mathrm{b}$ for $t^{\prime}$ and $t$, respectively), respectively. In these figures, in comparison to the SFA model, we also present the classical solutions of $t^{\prime}$ and $t$ (panels $\mathrm{c}$ and $\mathrm{d}$, respectively). From these figures it is clear that the SFA resembles the classical calculations. For the homogeneous case, the ionization and recombination times corresponding to each cycle are identical and the largest cutoff (harmonic $48 \omega)$ comes from the shortest pairs.
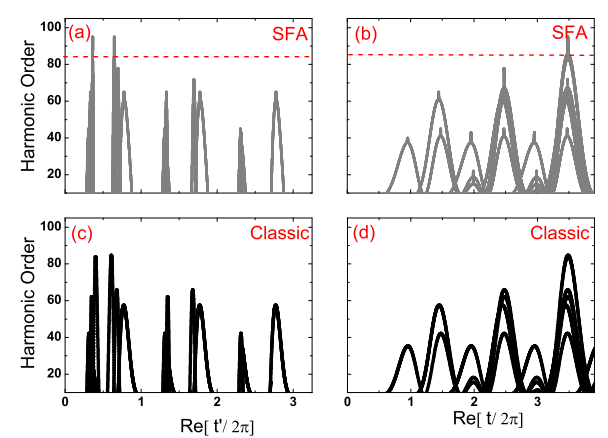

FIG. 3: (Color online) Dependence of harmonic order on the release time $t^{\prime}$ and the recombination time $t$ of the electron for a nonhomogeneous field with $\varepsilon=0.003$ using the same parameters as in Fig. 2 Panels (a) and (b) give the ionization and recombination times of SFA model, respectively, while panels (c) and (d) depict the ionization and recombination times of the classical calculations, respectively. The red dashed lines correspond to the harmonic cutoff.

For the nonhomogeneous case, the general harmonic cutoff is extended in comparison to the homogeneous case, but the trajectories do not follow the same symmetry as shown in Fig. 2

In here, the electron tunnels with two different canonical momenta given by Eq. (31): one leads to a higher cutoff, and the other to a lower one. For example, for the shortest pairs corresponding to the electron leaving at the field maxima the cutoff is at around harmonic $38 \omega$, while for the pairs corresponding to the electron leaving at the field minima it is at around harmonic $60 \mathrm{\omega}$.

Furthermore, the cutoff become larger as we move from shorter pairs to the longer pairs. As it demonstrated in Fig. 4 the trajectories from the minimum and maximum of the cycle moves towards each other until they collocate on each other at the field crossing. As $\varepsilon$ increases the solutions from positive and negative side of the cycle collapse faster to each other. It means there will be less available pairs of orbit for electron to produce HHG. In case of the field with very large $\varepsilon$ even the long trajectory of the shortest orbits are not allowed as 


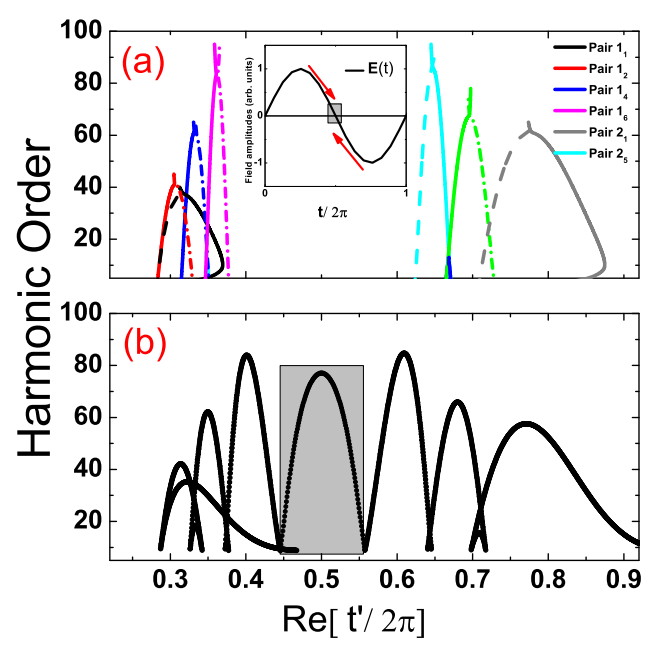

FIG. 4: (Color online) Harmonic order as a function of the release times $t^{\prime}$ of the electron for the same parameters given in Fig. 3. but for pairs corresponding to the solutions from positive and negative sides of the first cycle. Panel (a) gives the SFA real part of the release times, while panel (b) depict the classical ionization times of these pairs. The dashed and solid lines correspond to the long and the short orbits, while the pairs with dot dashed lines do not have the well-known shorts and long pairs.

we demonstrated it using the actual enhanced field of the nanostructure [32].

\section{E. Spectra}

In this section, we compute HHG spectra using Eq. (15).

These results are depicted in Fig. [5 with red colored spectra presenting the homogeneous field and blue colored and black colored spectra showing the nonhomogeneous case with $\varepsilon=0.003$ and $\varepsilon=0.005$, respectively. In all cases, the HHG cutoff is in good agreement with the trajectories analysis represented in the previous section and with the full 3D numerical calculations of Ref [12]. These cutoffs are at around harmonics $45 \omega, 73 \omega$ and $93 \omega$ for $\varepsilon=0, \varepsilon=0.003$ and $\varepsilon=0.005$, respectively. Moreover, it seems that both odd and even harmonics are present in the total spectra. At higher regime of the spectra, both odd and even harmonic have the same weight, while at the lower regime one sets of harmonics are a bit more dominant than the others.

\section{CONCLUSIONS AND OUTLOOK}

We present theoretical studies of high-order harmonic generation in noble gases produced by spatially nonho-

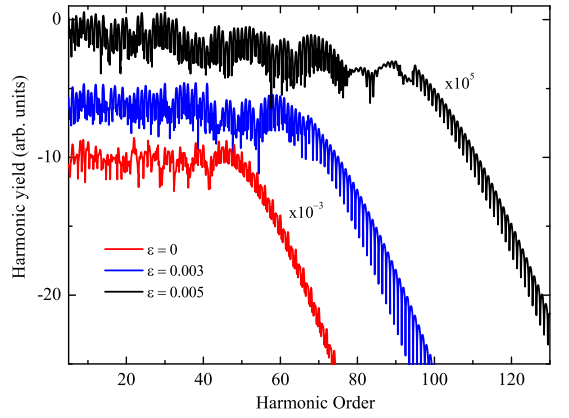

FIG. 5: (Color online) High-order harmonic spectra for hydrogen atoms $\left(I_{p}=0.5\right.$ a.u. $)$ interacting with a monochromatic field of frequency $\omega=0.057$ a.u. and intensity $I=$ $3 \times 10^{14} \mathrm{~W} / \mathrm{cm}^{2}$. Red, blue and black colors depict the cases with $\varepsilon=0, \varepsilon=0.003$ and $\varepsilon=0.005$, respectively. The HHG spectra obtained for $\varepsilon=0$ and $\varepsilon=0.005$ are scaled for clarity.

mogeneous fields. These fields are generated when different nanosystems, such as metal bow-tie nanostructures, metal and dielectric nanoparticles and nanotips, are exposed to a short and intense laser pulse. In this study, we have employed a numerical approach as well as performed an exhaustive theoretical analysis.

For the numerical method, we used the numerical solution of the time dependent Schroedinger equation (TDSE) in reduced dimensions combined with the actual functional form of the electric field extracted from finite element simulations to predict the harmonic spectra originated when Ar atoms interact with the plasmonic enhanced field in a metal bow-tie nanostructure. We observe an extension in the harmonic cutoff position that could lead to the production of XUV coherent laser sources and opening the avenue to the generation of attosecond pulses. For this particular nanostructure, this new feature is a consequence of the combination of a nonhomogeneous electric field, which modifies substantially the electron trajectories, and the confinement of the electron dynamics.

In our theoretical analysis, we employed both classical and semiclassical approaches to investigate the highorder harmonic spectra produced by spatially nonhomogeneous fields of linear form. We show how the quantum orbits manifest themselves in these particular spatially inhomogeneous fields. In addition we predict that in nonhomogeneous fields, the electron tunnels with two different canonical momenta leading to a higher and lower cutoff. We also conclude that in the case of linear nonhomogeneous fields, both odd and even harmonics are present in the HHG spectra. Within our model, we show that the HHG cutoff extends to the larger harmonics as a function of the inhomogeneity strength and this feature is consistent with the findings recognized using actual fields. 


\section{Acknowledgments}

We acknowledge the financial support of the MICINN project FIS2008-00784 TOQATA; ERC Advanced Grant QUAGATUA, Alexander von Humboldt Foundation and
Hamburg Theory Prize (M. L.). This research has been partially supported by Fundació Privada Cellex. We thanks Peter Hommelhoff and Samuel Markson for useful comments and suggestions.
[1] M. Protopapas, C. H. Keitel, and P. L. Knight, Rep. Prog. Phys. 60, 389 (1997).

[2] T. Brabec and F. Krausz, Rev. Mod. Phys. 72, 545 (2000).

[3] M. Lein, J. Phys. B 43, R135 (2007).

[4] F. Krausz and M. Ivanov, Rev. Mod. Phys. 81, 163 (2009).

[5] P. B. Corkum, Phys. Rev. Lett 71, 1994 (1993).

[6] M. Lewenstein, P. Balcou, M. Y. Ivanov, A. L'Huillier, and P. B. Corkum, Phys. Rev. A 49, 2117 (1994).

[7] S. Kim, J. Jin, Y.-J. Kim, I.-Y. Park, Y. Kim, and S.-W. Kim, Nature 453, 757 (2008).

[8] I.-Y. Park, S. Kim, J. Choi, D.-H. L. Y.-J. Kim, M. F. Kling, M. I. Stockman, and S.-W. Kim, Nat. Phot. 5, 677 (2011).

[9] P. Mühlschlegel, H.-J. Eisler, O. J. F. Martin, B. Hecht, and D. W. Pohl, Science 308, 1607 (2005).

[10] P. J. Schuck, D. P. Fromm, A. Sundaramurthy, G. S. Kino, and W. E. Moerner, Phys. Rev. Lett. 94, 017402 (2005).

[11] A. Husakou, S.-J. Im, and J. Herrmann, Phys. Rev. A 83, 043839 (2011).

[12] I. Yavuz, E. A. Bleda, Z. Altun, and T. Topcu, Phys. Rev. A 85, 013416 (2012).

[13] M. F. Ciappina, J. Biegert, R. Quidant, and M. Lewenstein, Phys. Rev. A 85, 033828 (2012).

[14] T. Shaaran, M. F. Ciappina, and M. Lewenstein, J. Mod. Opt. p. (in press) (2012).

[15] M. F. Ciappina, J. A. Pérez-Hernández, T. Shaaran, J. Biegert, R. Quidant, and M. Lewenstein, Phys. Rev. A 86, 023413 (2012).

[16] M. Sivis, M. Duwe, B. Abel, and C. Ropers, Nature 485, E1 (2012).

[17] S. Kim, J. Jin, Y.-J. Kim, I.-Y. Park, Y. Kim, and S.-W. Kim, Nature 485, E2 (2012).

[18] P. B. Corkum, (private communication) (2012).

[19] P. Hommelhoff, Y. Sortais, A. Aghajani-Talesh, and M. A. Kasevich, Phys. Rev. Lett. 96, 077401 (2006).

[20] M. Schenk, M. Krüger, and P. Hommelhoff, Phys. Rev. Lett. 105, 257601 (2010).

[21] M. Krüger, M. Schenk, and P. Hommelhoff, Nature 475,
$78(2011)$

[22] M. Krüger, M. Schenk, M. Förster, and P. Hommelhoff, J. Phys. B 45, 074006 (2012).

[23] G. Herink, D. R. Solli, M. Gulde, and C. Ropers, Nature 483, 190 (2012).

[24] P. Sailères, A. L'Huillier, P. Antoine, and M. Lewenstein, Advances in Atomic, Molecular and Optical Physics, eds. B. Bederson and H. Walther 41, 83 (1999).

[25] A. L'Huillier and M. Lewenstein, Strong Field Laser Physics, ed. T. Brabec Springer Series in Optical Sciences (Springer, Berlin, 2008).

[26] Q. Su and J. H. Eberly, Phys. Rev. A 44, 5997 (1991).

[27] J. L. Krause, K. J. Schafer, and K. C. Kulander, Phys. Rev. A 45, 4998 (1992).

[28] K. J. Schafer and K. C. Kulander, Phys. Rev. Lett. 78, 638 (1997).

[29] S. S. Aćimović, Introduction to nanoparticle characterization in COMSOL (available from http://srdjancomsol.weebly.com, 2011).

[30] P. B. Johnson and R. W. Christy, Phys. Rev. B 6, 4370 (1972)

[31] A. Thai, M. Hemmer, P. Bates, O. Chalus, and J. Biegert, Opt. Lett. 36, 3918 (2011).

[32] M. F. Ciappina, S. S. Aćimović, T. Shaaran, J. Biegert, R. Quidant, and M. Lewenstein, Opt. Express. (submitted), arXiv:1204.5911v1 (2012).

[33] F. Süßmann and M. F. Kling, Proc. of SPIE 8096, 80961C (2011).

[34] F. Süßmann and M. F. Kling, Phys. Rev. B 84, 121406(R) (2011).

[35] C. H. Edwards and D. E. Penney, Differential Equations and Boundary Value Problems - Computing and Modeling (Prentice Hall, 4th edition, 1999).

[36] W. Gordon, Z. Phys 40, 117 (1926).

[37] D. M. Volkov, Z. Phys 94, 250 (1935).

[38] P. Saliéres, B. Carre, L. L. Deroff, F. Grasbon, G. G. Paulus, H. Walther, R. Kopold, W. Becker, D. B. Milosevic, A. Sanpera, et al., Science 292, 902 (2001).

[39] T. Shaaran, M. F. Ciappina, and M. Lewenstein, Phys. Rev. A 86, 023408 (2012). 